\title{
Cuidado humanizado: un desafio para las enfermeras en los servicios hospitalarios
}

\author{
Humanized care: a challenge for nursing in the hospitals services \\ Cuidado bumanizado: um desafio para enfermeiras nos serviços hospitalares
}

Margarita Poblete Troncoso ${ }^{1}$, Sandra Valenzuela Suazo ${ }^{2}$

\section{RESUMEN}

El presente artículo es un análisis crítico de la influencia institucional en el trabajo de las enfermeras de los servicios de salud pública en Latinoamérica y como este afecta la visión humanista del cuidado. Un factor influyente son los sistemas institucionales impregnados del modelo biomédico donde las enfermeras realizan su trabajo lo que la ha llevado a una sobrecarga de trabajo y pérdida de su autonomía. Por otro parte, los usuarios valoran más los aspectos del cuidado que tienen relación con la comunicación, el afecto y la atención que se le presta dentro de las instituciones hospitalarias. Cuan importante es entonces que las enfermeras otorguen cuidados centrados en los usuarios y que los cuidados humanizados se integren a la praxis para mejorar la calidad de la atención en los servicios de salud.

Descriptores: Humanización de la atención; Hospitales; Atención de enfermería

\begin{abstract}
This article is a critical analysis of the institutional influence of nurses' work on public health care systems in Latin America, and on how this influence affects the humanistic view of nursing care. An important factor to be considered is that institutional health systems, where nurses work, are impregnated with the biomedical care model. The latter has led to a higher workload for nurses as well as loss of professional autonomy. On the other hand, users appreciate the aspects of healthcare associated with communication, affection, and the care they receive in the hospitals. Hence, it is crucial that nurses provide user-centered care. Moreover, humanized care should be incorporated to nursing practice in order to enhance the quality of public health care systems.
\end{abstract}

Keywords: Health care humanization; Hospitals; Nursing care

\section{RESUMO}

O presente artigo é uma análise crítica da influencia institucional do trabalho das enfermeiras nos serviços publico de saúde na América Latina e como este afeta a visão humanista do cuidado. Um importante fator é que os sistemas institucionais, onde as enfermeiras realizam seu trabalho, estão impregnados pelo modelo biomédico, que conduziu a uma sobrecarga de trabalho acompanhada pela perda da sua autonomia profissional. Entretanto, os usuários valorizam mais os aspectos do cuidado relacionados com a forma de comunicação, afeto e a assistência que lhes é prestada dentro das instituições hospitalares. É de suma importância então, que as enfermeiras outorguem cuidados centralizados nos usuários e que os cuidados humanizados se integrem na pratica, para melhorar a qualidade da assistência nos serviços de saúde.

Descritores: Humanização da assistência; Hospitais; Cuidados de enfermagem

\footnotetext{
1 Doctora do Programa de Doctorado en Enfermería, Universidad de Concepción, Concepción, Chile.; Profesora del Departamento de Enfermería, Facultad de Salud, Universidad Católica del Maule, Talca, Chile.

${ }^{2}$ Doctora, Profesora Asociada, Departamento de Enfermería, Facultad de Medicina, Universidad de Concepción, Concepción, Chile.
} 


\section{INTRODUCCIÓN}

El cuidado es la esencia de Enfermería, estos debieran estar constituidos por acciones transpersonales e intersubjetivas para proteger, mejorar y preservar la humanidad ayudando a la persona a hallar un significado a la enfermedad, sufrimiento, dolor y existencia y ayudar a otro a adquirir autocontrol, autoconocimiento y autocuración ${ }^{(1-2)}$.

Sin embargo las institucionales hospitalarias impregnadas del modelo biomédico y curativo, reduce lo humano a lo biológico alejando el trabajo de las enfermeras de su visión humanista y holistica del cuidado.

Las instituciones hospitalarias como subsistemas sociales, cumplen la función de socializar a los individuos con pautas, normas establecidas, adaptándolos e integrándolos al sistema, de esta forma el cuidado holistico se vea dificultado por las múltiples tareas delegados de tipo biomédica quedando relegado acciones como la comunicación eficaz y el interactuar con el paciente y familia en forma cercana denominado por Watson ${ }^{(1-2)}$ "cuidado transpersonal", dichas acciones valorizadas por los usuarios ${ }^{(3-5)}$.

\section{LAS INSTITUCIONES HOSPITALARIAS}

La institución hospitalaria como sistema social

Desde el nacimiento de la clínica a fines del siglo XVIII las instituciones hospitalarias, se constituyeron en centros de formación y saber médico, donde la patología pasa a ser su objeto de estudio perdiendo los enfermos su individualidad e integridad ${ }^{\left({ }^{(}\right)}$.

Desde entonces esta visión biologicista a impregnado el quehacer de las disciplinas que interactúan en las instituciones hospitalarias, Merhy ${ }^{(7)}$ refiere que esta hegemonía del modelo de acción clínica del médico termina por empobrecer y hasta anula las acciones cuidadoras del resto del equipo de salud.

Parsons $^{(8)}$ en su Teoría del Sistema Social se refiere a las instituciones como subsistemas sociales cuya función es socializar, cumpliendo la función de latencia, que significa la transmisión de patrones y pautas culturales, cuyo rol es controlar, normar y motivar a los individuos para mantener el sistema social integro.

De este modo, los subsistemas cumplen las siguientes funciones: adaptativa, socialización de pautas, integrativas hacia la sociedad, logros de fines.

De acuerdo a la Teoría de Parsons ${ }^{(9)}$, los subsistemas institucionales tienden a ser imperturbables en sus normas y logran en los individuos que ingresan a ellos, un control social como cualquier subsistema social, de tal forma que rápidamente los individuos se adaptan a sus normas y funcionamiento, es por ello que probablemente sea el aspecto institucional, el responsable de modificar el cuidado de enfermería en las institución de salud. El rol que enfermería cumple dentro de las instituciones hospitalarias esta socialmente estructurado, adquiriendo las profesionales como lo señala Watson ${ }^{(1)}$, una conducta institucionalizada.

La explicación de la conducta institucionalizada estaría según Parsons et al. ${ }^{(9)}$ en la Psicología de la Personalidad. La personalidad se desarrolla dentro de un sistema social y va actuar espontáneamente como prerrequisito funcional de los sistemas sociales de la que son parte, los individuos. Los individuos pasan a ser actores de estos subsistemas sociales cumpliendo un conjunto de roles y cada uno de sus roles está socialmente aceptado. Estos roles se van modelando y alienando en la interacción con otros individuos. Las acciones que los actores realizan dentro de un sistema social es un modo de cumplimiento de sus propias disposiciones de necesidades y a la vez una condición para "hacer óptimas" las reacciones de los otros actores significativos, de esta forma se puede decir que el criterio esta institucionalizado. Siempre existen dobles expectativas en las conductas de los actores en las instituciones, las que se refieren a sus propias expectativas de rol y las expectativas referentes a las reacciones contingentes probables de las otras, denominadas sanciones que pueden ser positivas o negativas es decir gratificaciones o privaciones. La personalidad humana es sensitiva frente a las actitudes de los otros, por lo cual su conducta va a ser premiada si esta conforme con el subsistema o castigada si se desvía del subsistema.

Es la instancia institucional en sí”, según Butelman ${ }^{(10)}$, la que determina las "fronteras, más o menos precisas, más o menos permeables entre el adentro y el afuera; la que decide sobre los individuos que la integran, sobre los extraños; recibe mandatos y demandas, genera proyectos, planes, programas; edifica una estructura organizativa, instala procedimientos y rutinas; favorece u obstaculiza procesos de cambios; genera mecanismos y modos de regulación de conflictos en un aparato jurídico -normativo",

Las instituciones hospitalarias, son subsistemas sociales que cumplen la función de socializar a los individuos con pautas, normas establecidas, adaptándolos e integrándolos al sistema con la finalidad de que cumplan sus roles establecidos a través de un control social, con el objetivo de mantener el sistema social imperturbable.

Merhy ${ }^{(7)}$ refiere que las instituciones de salud cumplen la función de otorgar cuidados a través de un trabajo vivo, y se espera de ellas que sean un núcleo de cuidado para los usuarios; sin embargo, el acto de cuidar es deficiente debido a que los diferentes profesionales que trabajan en los servicios de salud no realizan acciones de cuidado centrados en el paciente, sino centrados en los procedimientos o en la técnica, lo que actualmente ha provocado la crisis de estas instituciones, además se agrega 
el hecho de que la hegemonía del modelo de acción clínica del médico empobrece y hasta anula las acciones cuidadoras del resto del equipo de salud.

\section{El paradigma biomédico en la atención de salud}

Los aspectos biomédicos y curativos ${ }^{(11)}$ han tenido una influencia por siglos en los servicios de salud, el "curar" asumido por la medicina moderna, se funda en una perspectiva analítica, empírica y experimental, adecuada para el estudio de los procesos biológicos y fisiopátologicos, que, como fenómenos naturales, presentan estabilidad, regularidad, constancia y cierta independencia de contexto, pero, ha reducido lo humano a lo biológico; situación que viene desde el siglo XVIII, en que el cuerpo humano pasa a ser objeto específico de la aplicación de la medicina, y el enfermo pasa a ser una enfermedad.

El cuidado holistico en los servicios de salud se ha visto dificultado por esta visión biomédica de la salud, determinado que enfermería concibe la realidad dentro de los parámetros de la biomedicina, en donde el cuidado es concebido a partir de normas, expedientes, rutinas y patrones de conductas desde que las familias entran a los centros de salud ${ }^{(12-13)}$.

En Chile esta visión biomédica de la atención en salud en la cual se realizan los cuidados de enfermería, es lo que ha llevado a las enfermeras a una sobrecarga de trabajo de tipo biomédico y a labores en forma mecanizada, determinando que su trabajo se torne “invisible" en los campos clínicos ${ }^{(4)}$. Esta situación no es ajena en los servicios sanitarios brasileños en que el no reconocimiento a su labor, esta llevando a la insatisfacción profesional de las enfermeras/os, en que observan la desvalorización de su trabajo en los campos clínicos y el alejamiento de la humanización de los cuidados ${ }^{(14)}$.

Por otra parte, los usuarios de los centros de salud sienten la necesidad de que estos cuidados sean significativos, en donde el escuchar, la empatía y el afecto se consideren tan importante como los aspectos técnicos del quehacer enfermería ${ }^{(15)}$.

Sin embargo los profesionales de enfermería, como una forma de sentirse valorado han relacionado el cuidado con el accionar cotidiano (técnicas de enfermería) y consideran la tecnología como poder, orientando muchas veces su práctica hacia lo técnico distanciándose por tanto de los valores morales y políticos inserto en su trabajo cotidiano por otro lado son estos aspectos los que apartan a las enfermeras del análisis crítico ${ }^{(16)}$ de su realidad y la mantienen en una cómoda neutralidad en donde prevalece la rutinaria ejecución de procedimiento.

En Chile, si bien el estudio del cuidado, esta en una primera etapa se observa que las enfermeras tienen dificultad para concebir como propio la gestión del cuidado por su rol predominantemente biomédico, situación que es mirada con preocupación por el gremio de enfermeras profesionales en Chile ${ }^{(17)}$, " son las mismas enfermeras las que no conciben como propio la gestión del cuidado, observándose en las profesionales intra hospitalarias, que las actividades que realizan son en su mayoría derivadas del diagnóstico y tratamiento médico", a esto se agrega la limitación de su ejercicio de autonomía profesional en su quehacer diario.

Gutiérrez et al.(4), en su estudio del rol de enfermería observaron que las enfermeras presentaban solo satisfacción con actividades derivadas del diagnóstico y tratamiento médico (técnicas) y no de acciones que corresponden al proceso de atención de enfermería u otras del rol independiente.

Balanza $^{(3)}$ buscando el significado del cuidado de enfermería en los pacientes de los servicios hospitalarios, observa a través de su discurso que el cuidado para ellos se compone de preocupación, amor, ánimo, buen trato y atención, acción oportuna, habilitación, poder y dependencia, procesos que realizan los estudiantes de enfermería pero, es poco frecuente realizado por enfermeras profesionales.

\section{Visión humanista del cuidado de enfermería}

Los modelos y teorías de enfermería se fundamentan en una visión humanista del cuidado, como por ejemplo Watson $^{(1-2)}$, la cual refiere que el cuidado es para la enfermería su razón moral, no es un procedimiento o una acción, el cuidar es un proceso interconectado, intersubjetivo, de sensaciones compartidas entre la enfermera y paciente.

El cuidado humano debe basarse en la reciprocidad y debe tener una calidad única y auténtica. La enfermera es la llamada a ayudar al paciente a aumentar su armonía dentro de la mente, del cuerpo y del alma, para generar procesos de conocimiento de sí mismo. Desde este punto de vista, el cuidado no solo requiere que la enfermera sea científica, académica y clínica, sino también, un agente humanitario y moral, como copartícipe en las transacciones de cuidados humanos ${ }^{(1)}$.

A través de estudios transculturales Leininger ${ }^{(18)}$, realiza los primeros intentos para clarificar y conceptualizar la noción del cuidado: "el cuidado es para enfermería el dominio central del cuerpo de conocimiento y las prácticas". Afirma que, "el constructo cuidado se ha manifestado durante millones de años como fundamental en el crecimiento y supervivencia de los seres humanos". El cuidado permitió a la especie humana vivir y sobrevivir bajo las más adversas condiciones ambientales, sociales, económicas y políticas. Los antecedentes del cuidado ya se remontaban a la mitología romana, en que el "Cuidado" es quien crea al hombre y lo protege. De esto se deriva que la "acción de cuidar" es, entonces, algo propio del ser humano y revela su íntima constitución ${ }^{(19)}$.

Leininger ${ }^{(18)}$, considera que el cuidado es de carácter 
universal pero son los procesos, métodos y técnicas con los que se desarrollan los que varían culturalmente, es así que en la cultura anglosajona se distingue entre los términos de "care" y "caring", "care" es la acción de cuidar y "caring" incorpora además un sentido e intencionalidad humanitaria en enfermería.

Otro aspectos a considerar por teóricos de enfermería como Leininger es establecer la diferencia que existe entre curación y cuidados en donde agrega: "los procesos de curar y cuidar presentan diferencias en su esencia y en sus características principales; no puede existir curación sin cuidados pero puede existir cuidados sin curación" ${ }^{(18)}$.

Las diferencias ontológicas entre las actividades de curar y las de cuidar, de acuerdo a Medina ${ }^{(11)}$, se basan en la disimilitud de la perspectiva epistemológica y filosófica de la que ambas parten.

Los aspectos biomédicos y curativos se basan en una perspectiva analítica, empírica y experimental, reduciendo lo humano a lo biológico, el ser humano es una enfermedad u objeto de estudio.

En cambio, el "cuidado" de origen ancestral tiene una mirada diferente, ve al sujeto como enfermo- sujeto, antes que su enfermedad -objeto, permitiendo que el sufrimiento que puede ser aliviado no sea "ocultado" en la profundidad biológica del organismo. Los cuidados reducen las distancias entre los tratamientos y el sentido de la enfermedad, por lo cual enfermería se basa en una visión holistíca, biosicosocial, centrándose en las respuestas a los procesos mórbidos y de salud.

Otros autores como Colliere ${ }^{(20)}$, establecen que este saber ancestral de enfermería, ha estado oculto por la hegemonía que la racionalidad técnica, encarnada en el saber médico, que ha prevalecido en los sistemas de salud. De acuerdo a esta autora enfermería viene desarrollando tres tipos de actividades diferentes dentro de estas instituciones: las actividades que dependen directamente de la decisión e iniciativa de la enfermera en Chile esta definido en el código sanitario (Art.113) como rol independiente; las que dependen de la decisión médica y, las que dependen de iniciativas compartidas con el equipo de salud, estas últimas son conocidas como rol interdependiente (Art.113). Siendo las primeras de acuerdo a Colliere, las que han quedado difuminadas y ocultas en el tratamiento médico.

La visión humanista del cuidado de Watson ${ }^{(1)}$, es importante por cuanto cambia la visión de los cuidados de Enfermería en los sistemas hospitalarios, el cuidado debe adquirir una dimensión profunda que va más allá de una simple técnica, de planificar una atención, de recibir un turno o la de una educación rutinaria día a día; es estar ahí con el otro, es compartir sus sentimientos y sus emociones.

\section{CONCLUSIONES}

1. A modo de síntesis se observa en Latinoamérica la necesidad de buscar el significado que tiene el cuidado en los profesionales de enfermería y que los aspectos deficientes como es centrar el cuidado en el paciente y que este sea un "cuidado humanizado" se incorporen a la praxis.

2. Se colige entonces, que es el entorno inmediato, donde la enfermera desarrolla su trabajo, las instituciones hospitalarias, sistemas complejos, por cierto, con pautas y normas establecidas, el que de alguna forma están determinando que el acto de cuidar no sea centrado en el usuario.

3. Considerar la visión holistica y humanista del cuidado en nuestra disciplina como eje central en la formación de las estudiantes de enfermería, incorporando teorías de la comunicación transpersonal, considera claves en el cuidado.

4. Los estudios revelan que los pacientes valoran más los aspectos del cuidado que tienen relación con la comunicación, el afecto y la atención que se le presta dentro de las instituciones hospitalarias, es decir un cuidado humanizado y transpersonal.

5. Es difícil mantener los valores humanitarios en el acto de cuidar en las instituciones públicas de salud en donde parecen invisibilizarse los cuidados de enfermería por las labores biomédicas, pero las enfermeras deben mantener aún entonces las virtudes de comunicarse con el otro, ayudarlo y sostenerlo en esta difícil etapa de su vida.

\section{REFERÊNCIAS}

1. Watson J. Nursing: the philosophy and science of caring. Colorado: Colorado Associated University Press; 1985.

2. Watson J. Caring science as sacred science. Philadelphia: F.A. Davis Company; 2005.

3. Balanza N. El significado del cuidado de enfermería en la perspectiva del paciente adulto hospitalizado Cienc Enferm.1997; 3(1):59-68.

4. Gutiérrez González MP, Núñez Carrasco ER, Rivera CL. Características del rol en el profesional de enfermería intrahospitalario. Enfermería. 2002; 37(120): 29-33.

5. Rivas Riveros E. Posicionamiento de la enfermería: sustentabilidad y proyección. Enfermería. 2003; 38(124): 22-7.

6. Foucault M. El nacimiento de la clínica: una arqueología de la mirada médica. México: Siglo XXI; 1991.

7. Merhy EE. Saúde: a cartografia do trabalho vivo. São Paulo: Hucitec; 2002.

8. Parsons T. El sistema social. Madrid: Alianza; 1999.

9. Parsons T, Bales RF, Shils EA. Apuntes sobre la teoría de la acción. Buenos Aires: Amorrortu; 1970.

10. Bulteman I, compiladora. Pensando las instituciones, sobre teorías y prácticas en educación. Buenos Aires: Paidós; 1996. p. $140-61$.

11. Medina JL. La pedagogía del cuidado: saberes y prácticas en la formación universitaria en Enfermería. Barcelona: Laertes; 1999. p. 29-56. 
12. Monticelli M, Elsen I. El hospital como una "realidad clínica": una comprensión a partir del encuentro entre trabajadoras de enfermería y familias de un servicio de alojamiento conjunto. Horiz Enferm. 2004; 15:37-52.

13. Perpiña Galvan J. Análisis de los registros de enfermería del Hospital General Universitario de Alicante y pautas para mejorar su cumplimientación. Enferm Clín. 2005; 15(2):95-102.

14. Santos SR, Nóbrega MML. A busca da interação teoria e prática no sistema de informação em enfermagem: enfoque na teoria fundamentada nos dados. Rev Latinoam Enferm. 2004; 12(3):460-8.

15. Cardona L, Silva L. Relación entre la percepción de los comportamientos del cuidados de los pacientes y la del personal de enfermería de la unidad de cuidado intensivo del Hospital Santa Clara. El arte y la ciencia del cuidado. Grupo de cuidado, Facultad de Enfermería, Universidad Nacional de Colombia. (Trabajo de grado para optar el titulo de especialización en enfermería Cario Respiratória). Bogotá: Faculdad de Enfermería. Universidad Nacional de Colombia; 2004. p. 157-62.

16. Da Silva LF, Coelho M, De Lima C, Silva de Souza P. Cuidados de enfermería: su sentido para enfermeras y pacientes. Enfermería. 2002; 37(120): 22-8.

17. Colegio de Enfermeras de Chile, A.G. Autonomía en la gestión del cuidado de enfermería, eje temático 1. Enfermería. 2004; 39 (125): $11-6$.

18. Leininger M. Teoría de los cuidados culturales. In:: Marriner A, Raile M. Modelos y teorías de enfermería. 6a ed. Philadelphia: Mosby; 2007.p. 472-98.

19. Torralba F. Constructos éticos del cuidar. Enferm Intensiva. 2000; 11(3):136-41.

20. Collière MF. Promover la vida. De la práctica de las mujeres cuidadoras a los cuidados de enfermería. Madrid: Editorial McGraw-Hill Interamericana; 1993. 